\title{
Von Muscheln inspiriert
}

\section{Adrian Ritter}

Freischaffender Journalist

\author{
Miesmuscheln halten sich mit Proteinfäden am Meeresgrund fest. Forschende \\ wollen diese Eigenschaft für einen neuartigen Kleber nutzen, der verwundetes \\ Gewebe schliessen kann. Besonders interessant wäre dies für die Herzchirurgie.
}

Die Welle kommt, die Welle geht, die Muschel bleibt. Tagein, tagaus muss die Miesmuschel der Brandung standhalten, ohne den Halt an Felsen oder am Meeresgrund zu verlieren. Spiderman kann daneben einpacken, denn sein Spinnenfaden funktioniert zwar im Trockenen, nicht aber in feuchter Umgebung. Die Evolution hat der Miesmuschel ein ausgeklügeltes System beschert: Aus dem Fuss der Muschel wachsen Seidenfäden, die nicht nur hervorragend haften, sondern auch hochelastisch sind.

\section{Die Suche nach Anwendungen}

So erstaunt es nicht, dass sich die Wissenschaft zunehmend für die Eigenschaften der Miesmuschel interessiert und diese imitieren will. Zahlreiche Forschungsgruppen versuchen, die Haftfähigkeit der Miesmuschel nutzbar zu machen. Für die Medizin sind die Seidenfäden besonders interessant, weil darin zwei Strukturproteine perfekt zusammenspielen. Sie machen die Fäden nicht nur extrem haftbar, sondern sind auch biokompatibel.

"Bisher hat sich noch keine konkrete Anwendung aus der weltweiten Forschung ergeben", sagt Claudio Toncelli. Der Polymer-Chemiker will dies mit drei Forschungsgruppen der Eidgenössischen Materialprüfungs- und Forschungsanstalt (Empa) in St.Gallen und in Zusammenarbeit mit Forschenden der ETH Zürich ändern. Dazu wählen sie einen neuen Weg. Während sich viele andere Forschungsgruppen auf nur eine chemische Gruppe aus dem Muschelprotein fokussieren, will sich das Team um Toncelli das Zusammenspiel von zwei chemischen Gruppen aus beiden Proteinen zunutze machen. Toncelli fand im März 2020 statt. Er ist inzwischen nicht mehr an der Empa tätig. Das Forschungsprojekt wird unter der Leitung von Prof. René Rossi weitergeführt

adrianritter[at]gmx.ch schen Gruppen modifizieren lässt. Aus der Kombination dieser Stoffe hofft das Team jetzt einen extrem haftbaren Gewebekleber herstellen zu können.

Der Hintergrund: Gelatine wird aus Kollagen hergestellt. Das wäre als Basis für einen medizinisch verwendbaren Gewebekleber sehr attraktiv, weil Kollagen ein natürliches Polymer und damit biokompatibel ist. Der Nachteil von Gelatine ist, dass sie bei Körpertemperatur nicht stabil ist, sondern sich verflüssigt. In Kombination mit den beiden modifizierten chemischen Gruppen lässt sich das Problem lösen: Sie verhindern, dass die Gelatine flüssig wird.

Der neuartige Kleber wird gemäss Toncelli für alle Gewebe nutzbar sein, die Kollagen enthalten - etwa Bindegewebe, Muskeln, Sehnen, Blutgefässe und Haut. Hier kann der Superleim helfen, Löcher im Gewebe zu schliessen.

\section{Fokus auf den Herzmuskel}

Das Forschungsteam hat bereits Anfragen von Medizinerinnen und Medizinern aus verschiedenen Fachbereichen erhalten. Vorerst fokussiert sich die weitere Forschung auf die Anwendung am Herzmuskel. Hier kämen die Vorteile des Superklebers am besten zur Geltung. Da er sich innerhalb von nur 30 Sekunden stabil mit dem Gewebe verbindet, eignet er sich besonders gut, um etwa Löcher im Herzen zu schliessen - ein Organ, das ständig in Bewegung ist. Davon könnten beispielsweise Neugeborene mit einem Ventrikelseptumdefekt profitieren. Die bisherigen Laborexperimente verliefen erfolgversprechend. «Der Gewebekleber hält einem Druck stand, der dem menschlichen Blutdruck entspricht», sagt Kongchang Wei. Zudem konnten die Forschenden die gute Gewebeverträglichkeit des neuen Klebers in Zellkultur-Experimenten bestätigen. Bis zu einer klinischen Anwendung wird es allerdings noch dauern. Vorerst sind weitere Studien nötig, etwa zur Biodegradabilität des Klebers. Das Forschungsteam der Empa bleibt dran. Wie die Miesmuscheln. 